\title{
Secondary Brain Lymphoma in a Case of Breast Diffused Large B-Cell Lymphoma: Case Report
}

\section{Hamed Javadian*, Reza Jalili Khoshnood, Mohammad Reza Shahmohammadi, Seyed Amir Hassan Hosseini, Mohsen Keikhaee}

Functional Neurosurgery Research Center, Shohada Tajrish Neurosurgical Comprehensive Center of Excellence, Shahid Beheshti University of Medical Sciences, Tehran, Iran

\begin{abstract}
Secondary central nervous system lymphoma (SCNSL) is known as a rare disease. The risk factor of developing SCNSL is primary lymphoma type and site of involvement. We present a patient with an altered mental status known case of breast diffused large B-cell lymphoma (DLBCL) who underwent stereotactic biopsy because of a left periventricular mass lesion, which diagnosed as secondary brain lymphoma after pathologic typing. Because of limited data about the secondary central nervous system, lymphoma and it is a risk factor, we reported an aggressive breast DLBCL with brain involvement.
\end{abstract}

\section{*Correspondence to}

Hamed Javadian, Functional

Neurosurgery Research Center, Shahid Beheshti University of Medical Sciences, Tehran, Iran

Email: Hamed.javadian@gmail.com Published online 30 September 2018

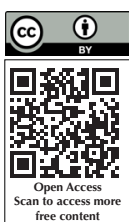

Citation: Javadian H, Jalili Khoshnood R, Shahmohammadi MR, Hosseini SAH, Keikhaee M. Secondary brain lymphoma in a case of breast diffused large B-cell lymphoma: case report. Int Clin Neurosci J. 2018;5(3):113-114. doi:10.15171/icnj.2018.22

\section{Introduction}

Secondary central nervous system lymphoma (SCNSL) is a lymphoma not derived from central nervous system (CNS) and can be a recurrence in the CNS or a part of a progressive disease. ${ }^{1}$ It is typically a non-Hodgkin lymphoma. SCNSL divided into subtype, leptomeningeal and parenchymal, or as a combination of this two type. Leptomeningeal lymphoma accounts for two-thirds of cases of secondary CNS lymphomas, the one-third remaining is parenchymal disease. ${ }^{2}$ because of the lack of clinical data about isolated SCNSL, a standard treatment regimen, and overall prognosis have not identified. ${ }^{3}$

\section{Case Presentation}

A 61-year-old woman with confusional state and amnesia admitted in our hospital. The patient had muscle weakness, right side paresis and was ill but not toxic. She had a medical history of breast lymphoma type diffused large B-cell lymphoma (DLBCL) 18 months ago, which treated with chemotherapy and radiotherapy. The patient family history was unremarkable. HIV test was negative. A metastasis workup revealed no other site of involvement. She had three recurrences despite treatment which means the aggressive type of disease. On brain magnetic resonance imaging (MRI) she had a mass lesion $(43.34 .38 \mathrm{~mm})$ on left frontal and periventricular region with peripheral edema, enhanced on $\mathrm{T} 1+\mathrm{GAD}$ sequence and restricted on diffusion weighted imaging (DWI), compatible with high-grade glioma and lymphoma.

The patient underwent a stereotactic biopsy. The result of biopsy was malignant high-grade B-CELL lymphoma after CD marker typing, diagnosed as SCNSL.

The patient received a high dose of methotrexate chemotherapy and planed for whole brain radiotherapy.

\section{Discussion}

The incidence of SCNSL depends on the type of primary lymphoma, the involvement of more than one site, stage of the primary disease and grade of the International Prognostic Index. ${ }^{1,2}$ patient with involvement of the breast, testis, and bone marrow as the primary disease is at a higher risk of developing SCNSL. ${ }^{4}$ The immunodeficient population are at the highest risk. Because of better diagnostic and treatment approach of primary and secondary CNS lymphoma, focusing more study on
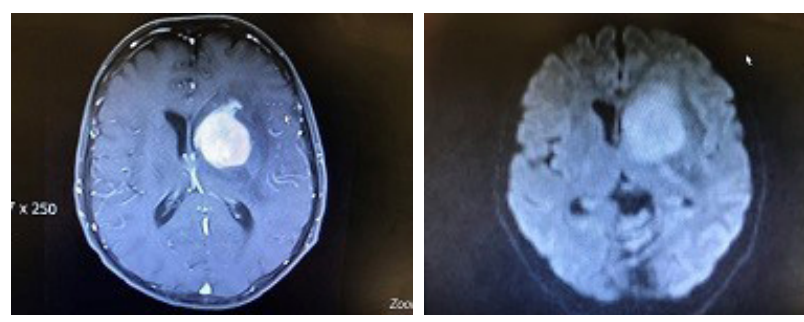

Figure 1. T1+GAD MRI imaging show enhancement and restricted on DWI. 
treatment of the elderly patient is needed. ${ }^{5}$

As noted the histologic grade of the primary lymphoma relate to developing of SCNSL. Up on the primary lymphoma classification, indolent, aggressive, or highly aggressive, there is a $3 \%, 9 \%$, and $27 \%$ risk of developing SCNSL. Especially for diffuse large B-cell lymphoma (DLBCL), the incidence has reported 5\%, but when the primary lymphoma is the mediastinal large B-cell type, the risk of SCNSL raise to $19 \%$. Indolent lymphomas have a low risk of recurrence, but when CNS involvement has proved, it is usually after the histologic transformation to a more aggressive type. ${ }^{3,6}$

Treatment method and CNS prophylaxis regime after the diagnose of primary lymphoma is a field of excellent research. Large retrospective trial studies found that an intravenous (IV) rituximab added to cyclophosphamide, doxorubicin, vincristine, and prednisolone (CHOP) therapy has decreased the incidence of SCNSL from $6.9 \%$ to $4.1 \%{ }^{7}$

The use of IV MTX has increased survival times for isolated SCNSL, especially for parenchymal SCNSL. ${ }^{7}$

\section{Conflict of Interest Disclosures}

The authors declare that they have no conflict of interests.

\section{Ethical Statement}

Informed consent was obtained form the patient for publication of this report.

\section{References}

1. Tomita N, Kodama F, Kanamori H, Motomura S, Ishigatsubo Y. Secondary central nervous system lymphoma. Int J Hematol. 2006;84(2):128-35. doi: 10.1532/ijh97.06091.

2. Feugier P, Virion JM, Tilly $H$, Haioun C, Marit G, Macro $M$, et al. Incidence and risk factors for central nervous system occurrence in elderly patients with diffuse largeB-cell lymphoma: influence of rituximab. Ann Oncol. 2004;15(1):129-33.

3. van Besien K, Gisselbrecht C, Pfreundschuh M, Zucca E. Secondary lymphomas of the central nervous system: risk, prophylaxis and treatment. Leuk Lymphoma. 2008;49 Suppl 1:52-8. doi: 10.1080/10428190802311458.

4. Kridel R, Dietrich PY. Prevention of CNS relapse in diffuse large B-cell lymphoma. Lancet Oncol. 2011;12(13):1258-66. doi: 10.1016/s1470-2045(11)70140-1.

5. Roth P, Martus P, Kiewe P, Mohle R, Klasen H, Rauch M, et al. Outcome of elderly patients with primary CNS lymphoma in the G-PCNSL-SG-1 trial. Neurology. 2012;79(9):890-6. doi: 10.1212/WNL.0b013e318266fcb2.

6. Colocci N, Glantz M, Recht L. Prevention and treatment of central nervous system involvement by non-Hodgkin's lymphoma: a review of the literature. Semin Neurol. 2004;24(4):395-404. doi: 10.1055/s-2004-861534.

7. Boehme V, Zeynalova S, Kloess M, Loeffler M, Kaiser U, Pfreundschuh $M$, et al. Incidence and risk factors of central nervous system recurrence in aggressive lymphoma--a survey of 1693 patients treated in protocols of the German HighGrade Non-Hodgkin's Lymphoma Study Group (DSHNHL). Ann Oncol. 2007;18(1):149-57. doi: 10.1093/annonc/ mdl327. 